\title{
Localized Immunoglobulin Light-Chain Amyloidosis of the Ulnar Nerve
}

\author{
Shinsuke Morisaki ${ }^{a} \quad$ Shinji Tsuchidab ${ }^{b}$ Eiichi Konishic Nagaaki Katoh ${ }^{d}$ \\ Yusuke Takahashi $^{d}$ Kenji Takahashi $^{\text {b }}$ \\ aDepartment of Orthopaedics, Saiseikai Shiga Hospital, Ritto, Japan; bepartment of \\ Orthopaedics, Graduate School of Medical Science, Kyoto Prefectural University of \\ Medicine, Kamigyo-ku, Japan; 'Department of Surgical Pathology, Graduate School of \\ Medical Science, Kyoto Prefectural University of Medicine, Kamigyo-ku, Japan; dDepartment \\ of Medicine (Neurology and Rheumatology), Shinshu University, Matsumoto, Japan
}

\section{Keywords}

Amyloid · Ulnar nerve · Neuropathy

\begin{abstract}
Amyloidosis is a disorder caused by extracellular tissue deposition of insoluble fibrils. Amyloidosis can be divided into systemic or localized disease. Primary systemic amyloidosis is a multisystem disease caused by the deposition of amyloid in various tissues. Localized amyloidosis has different characteristics than those of systemic amyloidosis. In this paper, we present the case of a middle-aged woman who presented with worsening ulnar nerve palsy. Electrophysiological examination and MRI indicated a tumor surrounding the ulnar nerve in the forearm. However, the operative findings revealed that ulnar nerve fascicles were replaced with a yellow tissue, which was diagnosed as amyloid light-chain $\lambda$ amyloidosis, based on histopathological examination. Systemic amyloidosis was ruled out after the screening examinations. This paper is the first report of the ulnar nerve as the sole site of localized immunoglobulin light-chain amyloidosis manifestation.
\end{abstract}

\section{Introduction}

Amyloidosis is characterized by extracellular fibrillar protein deposits that display Congo red staining and pathognomonic birefringence under cross-polarized light related to the $\beta$-sheet content [1].The 4 most common types of amyloids are immunoglobulin light chain $(\mathrm{AL})$, amyloid A (AA), transthyretin, and amyloid-beta peptide [2]. Immunoglobulin light- 
chain amyloidosis is characterized by a clonal population of bone marrow plasma cells that produces a monoclonal light chain of $\kappa$ and $\lambda$ type as an intact molecule or as a fragment [3].

Amyloidosis can be systemic or localized. Primary systemic amyloidosis is a multisystem disease caused by the deposition of amyloid in various tissues such as kidneys, cardiac, peripheral nerves, and musculoskeletal tissues. Approximately 15\% of patients develop peripheral neuropathy, which may be the major manifestation or may be overshadowed by other evidence of amyloidosis [4].

However, localized amyloidosis has a different character from that of systemic amyloidosis. Localized amyloidosis has never been known to progress systemically. Localized amyloidosis commonly occurs in the upper airway (e.g., nasopharynx and tongue); orbits; urinary tract, including the urinary bladder; and musculoskeletal tissues (e.g., skin and nails) [5]. We present the first report of localized amyloid neuropathy of the ulnar nerve in which the nerve fascicles were replaced by amyloid deposits and for which transplantation was performed.

\section{Case Report/Case Presentation}

A 54-year-old female patient presented to our clinic with complaints of occasional pain, numbness, and paralysis of her right hand. She began to notice weakness of her left hand that had begun 2 years earlier. She had no history of trauma and no family history of amyloid neuropathy. She had a history of ovarian tumor 25 years ago and thyroid tumor 5 years ago, both of which healed after surgical resection.

Physical examination revealed a mass $5 \times 3 \mathrm{~cm}$ in the ulnar side of her left proximal forearm shown in Figure 1. The lesion was firm, nonpulsatile, and tender on palpitation. She had numbness on the ulnar side of her left hand and fingers. The 2-point discrimination test revealed that $15 \mathrm{~mm}$ at the lateral side of the ring finger $10 \mathrm{~mm}$ at the little finger. She had muscle weakness with abduction and adduction of the ring finger and the little finger, but no deficit in the range of motion in the elbow. The electrophysiological study revealed a significant decrease in the motor nerve conduction velocity of the ulnar nerve from distal to the mass to proximal to the mass. MRI revealed that the ulnar nerve was swollen longitudinally, had a spindle shape, and measured $3.0 \times 2.7 \mathrm{~cm}$ in the axial plane and $7.2 \mathrm{~cm}$ longitudinally shown in Figure 2. The swollen lesion had a relatively homogeneous isosignal intensity on T1-weighted images and had relative heterogeneous enhancement after the intravenous administration of gadolinium (Gd)-based contrast agent with the findings of flow void. Based on these findings, the differential diagnoses included a vascular malformation that surrounded and compressed the ulnar nerve. Excisional biopsy was not performed because of the risk of

Fig. 1. The macroscopic view of the right forearm shows swelling in the medial proximal forearm on the marked area with black dots (arrow).

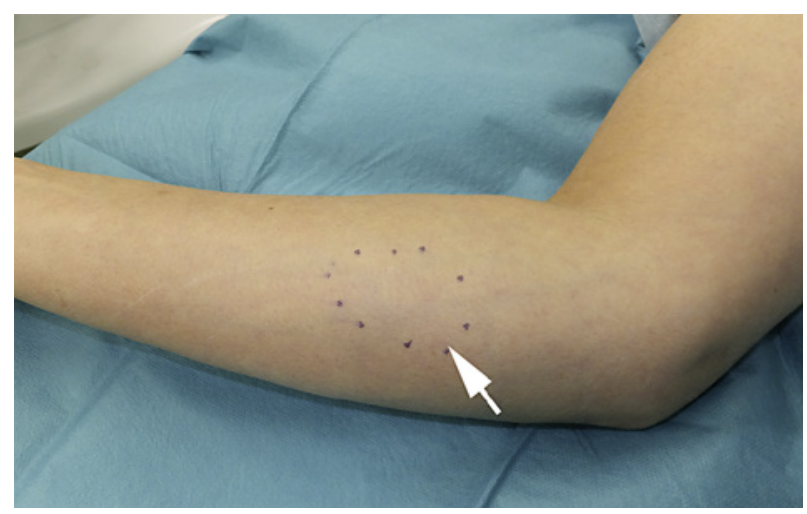



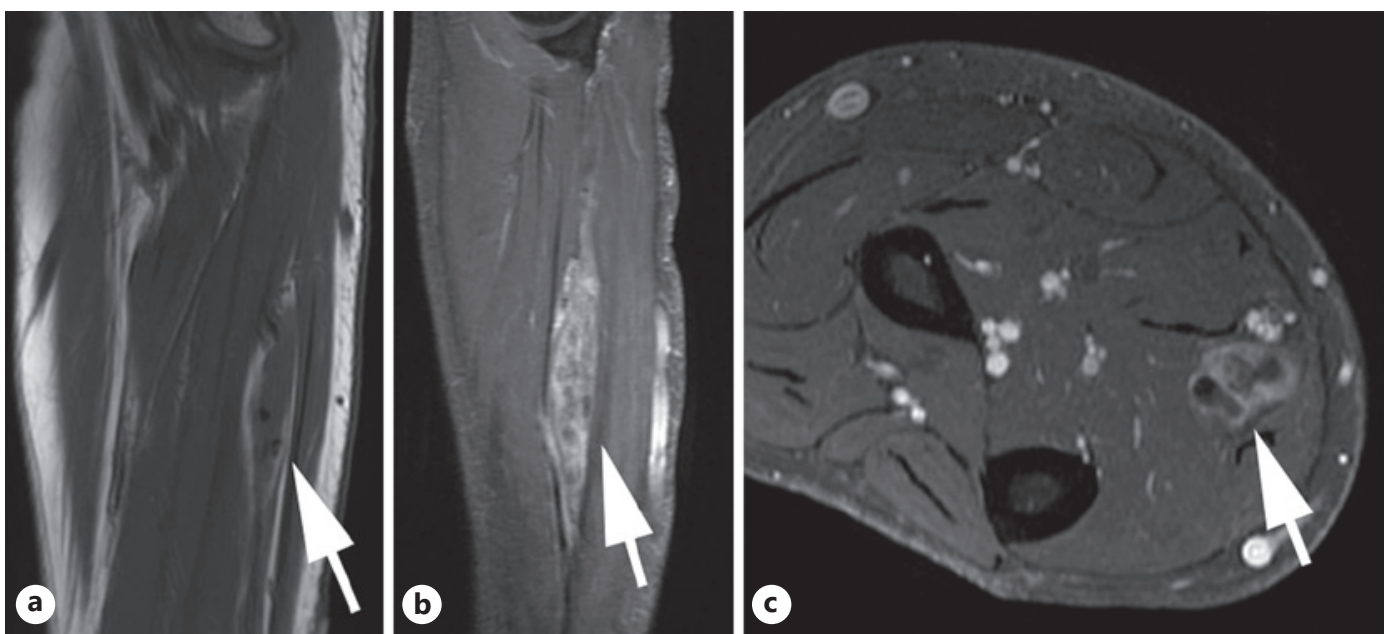

Fig. 2. MRI of the right forearm shows (a) the ulnar nerve is swollen with a low-signal intensity on T1-weighted images and (b) heterogeneously high-signal intensity on Gd-enhanced T1-weighted images in the sagittal view. The axial view shows flow voids (arrow) (c). Gd, gadolinium.
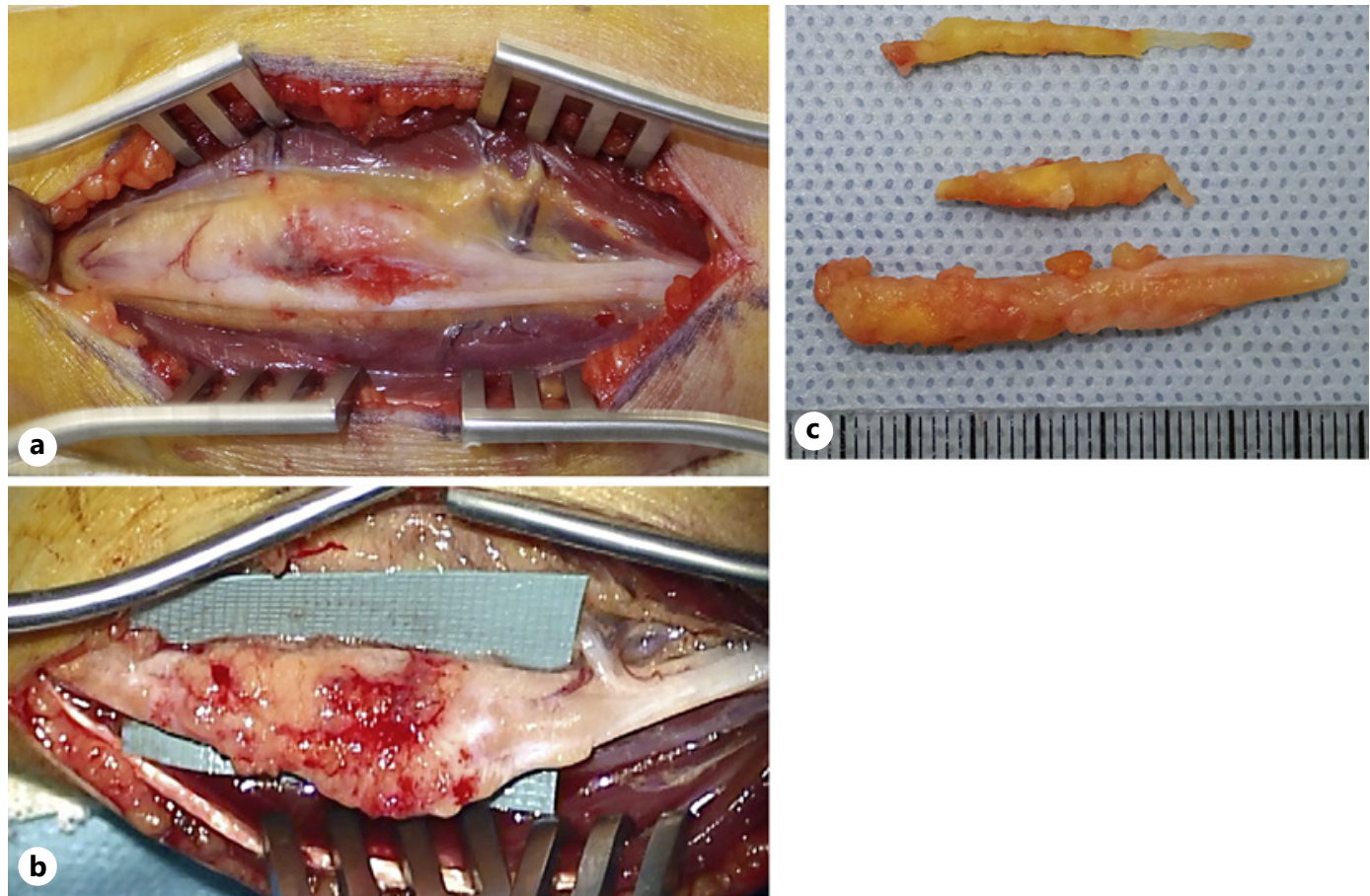

Fig. 3. The macroscopic view of the ulnar nerve. The right side is the proximal part. a The central part is swollen and the nerve fascicles are spread with slight hemorrhaging. $\mathbf{b}$ The nerve fascicles are degenerated and replaced with a yellow tissue. c The resected tissue.

hemorrhage and iatrogenic nerve injury. The lesion was instead surgically explored with a longitudinal incision shown in Figure 3a. The operative findings showed no vascular malformation; however, the ulnar nerve fascicles were swollen and degenerated. The affected lesion had changed into a yellow tissue shown in Figure 3b. After the yellow tissue was carefully removed microscopically, approximately $7 \mathrm{~cm}$ defects remained between intact nerve fascicles. The resected tissue was hard and had a stick-like shape shown in Figure 3c. 
The histopathological examination showed degenerated connective tissue with diffuse eosinophilic deposition, which included calcification, ossification, and chondroid tissue shown in Figure $4 \mathrm{a}$ and $\mathrm{b}$. No intact nerve tissue remained. The deposit was positive for Congo red stain and appeared apple-green under polarized light, which suggested the diagnosis of amyloid neuropathy shown in Figure 4c.

Immunohistochemical staining showed that the deposit was immunopositive for lightchain $\lambda$ antibody shown in Figure $4 \mathrm{~d}$. The deposit was immunonegative for amyloid A, transthyretin, and light-chain $\kappa$. Therefore, the pathological diagnosis was AL amyloidosis.

To determine the association with systemic involvement, we conducted screening examinations. Bone marrow aspiration, cerebrospinal fluid examination, echocardiography, electromyography of the legs, chest computed tomography, and MRI of the brain and legs showed negative results. Physical examination revealed no sign of macroglossia or purpura on the skin. Autonomic dysfunction, including orthostatic hypotension, was not present. The only positive results were from the blood test, which showed high serum levels of amyloid protein A $(159.2 \mu \mathrm{g} / \mathrm{mL}[<8.0])$.

Two months postoperatively, the patient underwent nerve transplantation for reconstruction of the ulnar nerve. The sural nerve was sacrificed from the left lower leg and transplanted to the defect of the ulnar nerve. One year postoperatively after the nerve
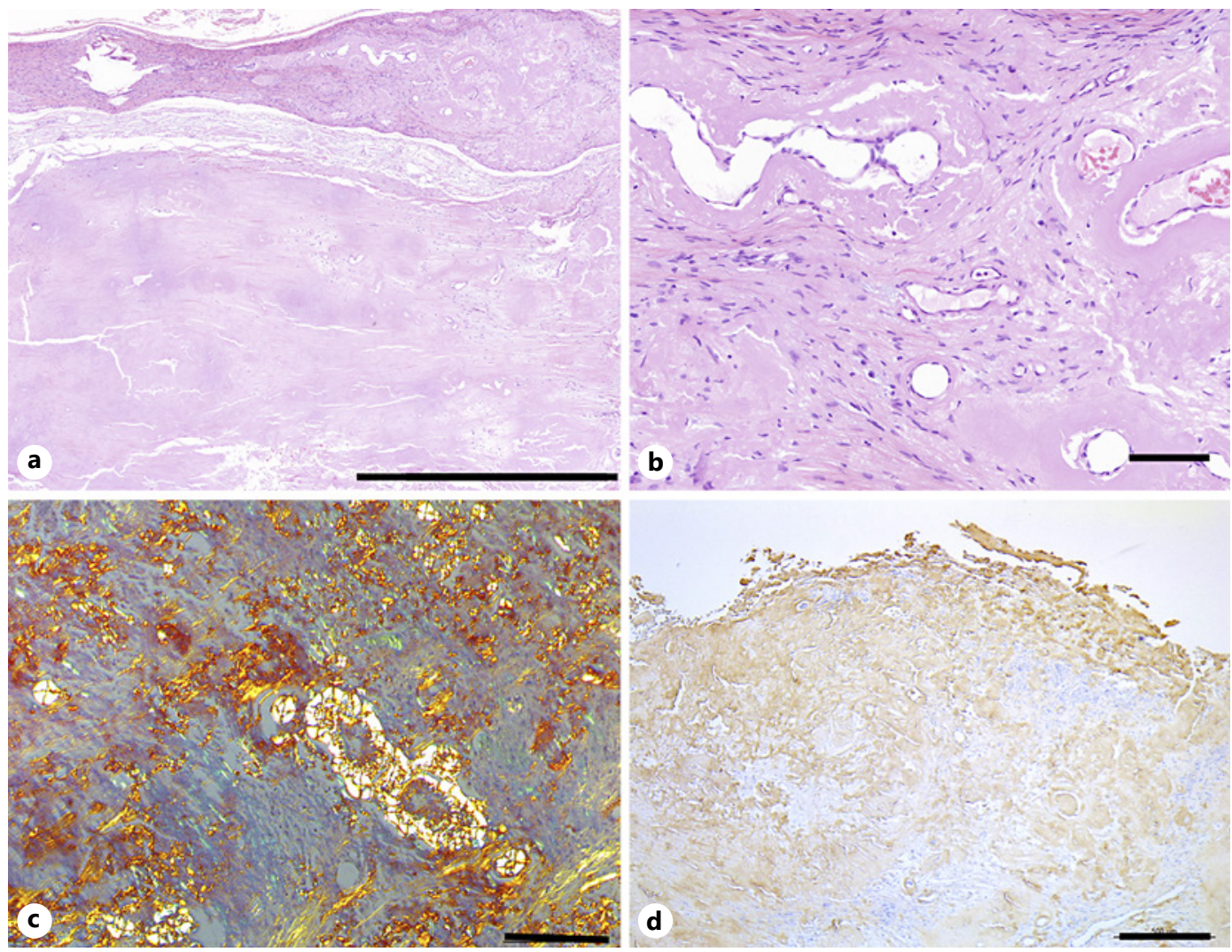

Fig. 4. Histological examination. $H \& E$ staining shows that the tissue includes degenerated tissue such and bone, chondroid tissue, and hair with no remaining intact nerve fibers. The overview image (the bar $=2,000$ $\mu \mathrm{m})(\mathbf{a})$ and the close-up image (the bar $=100 \mu \mathrm{m}$ ) (b). c Congo red stain appears apple-green under polarized light, which suggests the diagnosis of amyloid neuropathy (the bar $=2,000 \mu \mathrm{m}$ ). $\mathbf{d}$ Immunohistochemical staining shows that the deposit is immunopositive for light-chain $\lambda$ antibody (the bar $=500 \mu \mathrm{m}$ ). H\&E, hematoxylin and eosin. 
transplantation, she had no apparent evidence of metastasis or local recurrence. There was no recovery of numbness in the ring and little finger and the muscle weakness still had no recovery.

\section{Discussion/Conclusion}

Amyloidosis localized to 1 tissue is not uncommon. However, the ulnar nerve as the sole site of localized AL amyloidosis manifestation has never been reported in the literature.

Reports of localized AL amyloidosis of a peripheral nerve are extremely rare. AL amyloid deposition can occur at virtually any site of the body, including the brain [5]. The most common sites are eyelids, larynx, bronchi, skin, and urinary tract [6]. In a previous report, no cases of localized amyloidosis among 35 French patients involved the peripheral nerve [1]. Cheng reported a case of amyloidosis of the radial nerve in which an intraneural mass was bordered by stretched radial nerve fascicles [7]. The mass was excised via intracapsular decompression. The patient's motor function improved postoperatively. However, in our patient, amyloid was dominant in the ulnar nerve fascicles, and few intact nerve fascicles remained. Thus, local progression appears to have been advanced before the patient visited our clinic.

To diagnose localized amyloidosis, ruling out the possibility of systemic amyloidosis is essential $[4,8,9]$. The frequency of neuropathy in primary systemic amyloidosis is between 15 and 20\% [9]. Therefore, to distinguish localized amyloidosis from systemic amyloidosis, we conducted screening examinations, which revealed no signs of the involvement of the heart, kidney, liver, and peripheral nerves - except for the affected ulnar nerve. Bone marrow aspiration showed no evidence of multiple myeloma. Therefore, we diagnosed the patient as having localized AL amyloidosis solely of the ulnar nerve.

She had a history of ovarian tumor and thyroid tumor although she had not been diagnosed as having amyloidosis when she was previously treated. Furthermore, the ovaries and thyroid have not been included in cases of organ involvement in primary systemic amyloidosis [9]. Thus, her history was not correlated with amyloidosis of the ulnar nerve.

Histopathological examination was important for diagnosing amyloidosis. The resected tissue was soft and yellow and appeared to be fat-like rather than like nerve fascicles. Hematoxylin and eosin staining revealed only degenerated connective tissue, including a calcified structure, which did not indicate amyloidosis. Congo red staining revealed the typical faint apple-green birefringence under a polarized light source, which suggested amyloidosis. To identify the type of amyloid, the tissue underwent immunofluorescent staining, which showed that the deposits were immunopositive for the light-chain $\lambda$ antibody. AL amyloidosis was consequently diagnosed.

The long-term prognosis of localized amyloidosis is usually favorable, compared to systemic amyloidosis, which is usually lethal. For systemic amyloidosis, administering chemotherapy to suppress plasma cell clonal expansion is suggested. However, for localized amyloidosis, treatment is generally confined to local surgical intervention and resection [5]. In our patient, the portion of the ulnar nerve affected by amyloid was resected. After the resection, the nerve had a long defect approximately $7 \mathrm{~cm}$ long. Therefore, we performed reconstruction surgery by using the sural nerve for transplantation. One year postoperatively, the patient had no apparent motor and sensory recovery. However, no sign of the aggravation of symptoms occurred [8].

For our patient, diagnosing amyloidosis in the preoperative examinations was difficult. Except for the affected ulnar nerve, no signs of abnormalities existed. The MRI revealed a mass that involved the ulnar nerve. The mass was suspected of being a vascular malformation

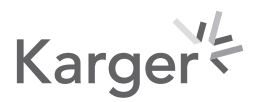


because the swollen lesion showed relative heterogeneous enhancement after the intravenous administration of a Gd-based contrast agent with the findings of flow void. If the mass were a vascular malformation, and then a needle biopsy should not be performed because of the risk of hemorrhage. Therefore, operative resection was the sole procedure to resolve the ulnar nerve palsy. In the preoperative examinations, our MRI findings indicating heterogeneous signal changes in Gd-enhanced condition was important to suspect amyloidosis of the nerve.

In this paper, we reported localized AL amyloidosis of the ulnar nerve, which has never been reported. The affected nerve fascicles were replaced with amyloid deposition and required nerve transplantation for treatment. The MRI findings of heterogeneous high-signal changes can be a diagnostic sign for indicating amyloidosis of a nerve.

\section{Acknowledgements}

The authors are grateful to Dr. Akihiro Fujii and Dr. Kenichi Kitamura for performing several examinations for diagnosis and technical suggestions.

\section{Statement of Ethics}

Written informed consent was obtained from the patient for publication of this case report and any accompanying images.

\section{Conflict of Interest Statement}

All the authors have no conflicts of interest to disclose.

\section{Funding Sources}

All the authors received no financial support for the research, authorship, and publication of this article.

\section{Author Contributions}

S.M. wrote the manuscript and prepared the pictures. S.T. and K.T. contributed to conceiving and designing the study and critically revised the manuscript. E.K., N.K., and Y.T. performed the pathological examinations. All the authors read and approved the final manuscript.

\section{References}

1 Paccalin M, Hachulla E, Cazalet C, Tricot L, Carreiro M, Rubi M, et al. Localized amyloidosis: a survey of 35 French cases. Amyloid. 2005 Dec;12(4):239-45.

2 Merlini G, Comenzo RL, Seldin DC, Wechalekar A, Gertz MA. Immunoglobulin light chain amyloidosis. Expert Rev Hematol. 2014 Feb;7(1):143-56.

3 Gertz MA. Immunoglobulin light chain amyloidosis: 2018 update on diagnosis, prognosis, and treatment. Am J Hematol. 2018 Sep;93(9):1169-80.

\section{Karger's}


4 Kelly JJ Jr, Kyle RA, O’Brien PC, Dyck PJ. The natural history of peripheral neuropathy in primary systemic amyloidosis. Ann Neurol. 1979 Jul;6(1):1-7.

5 Westermark P. Localized AL amyloidosis: a suicidal neoplasm? Ups J Med Sci. 2012 May;117(2):244-50.

6 Fuah KW, Lim CTS. Renal-limited AL amyloidosis: a diagnostic and management dilemma. BMC Nephrol. 2018 Nov 6;19(1):307.

7 Cheng RR, Eskandari R, Welsh CT, Varma AK. A case of isolated amyloid light-chain amyloidosis of the radial nerve. J Neurosurg. 2016 Sep;125(3):598-602.

8 Matsuda M, Gono T, Morita H, Katoh N, Kodaira M, Ikeda S. Peripheral nerve involvement in primary systemic AL amyloidosis: a clinical and electrophysiological study. Eur J Neurol. 2011 Apr;18(4):604-10.

9 Rajkumar SV, Gertz MA, Kyle RA. Prognosis of patients with primary systemic amyloidosis who present with dominant neuropathy. Am J Med. 1998 Mar;104(3):232-7. 